PROFESIONALES Y HERRAMIENTAS PARA EL DESARROLLO LOCAL Y SUS SINERGIAS TERRITORIALES. EVALUACIÓN Y PROPUESTAS DE FUTURO IX Coloquio Nacional de Desarrollo Local del GTDL-AGE 

ANTONIO MARTÍNEZ PUCHE, XAVIER AMAT MONTESINOS, ISABEL SANCHO CARBONELL y DANIEL SANCHIZ CASTAÑO (EDS.)

\section{PROFESIONALES Y HERRAMIENTAS PARA EL DESARROLLO LOCAL Y SUS SINERGIAS TERRITORIALES. EVALUACIÓN Y PROPUESTAS DE FUTURO}

IX Coloquio Nacional de Desarrollo Local del GTDL-AGE

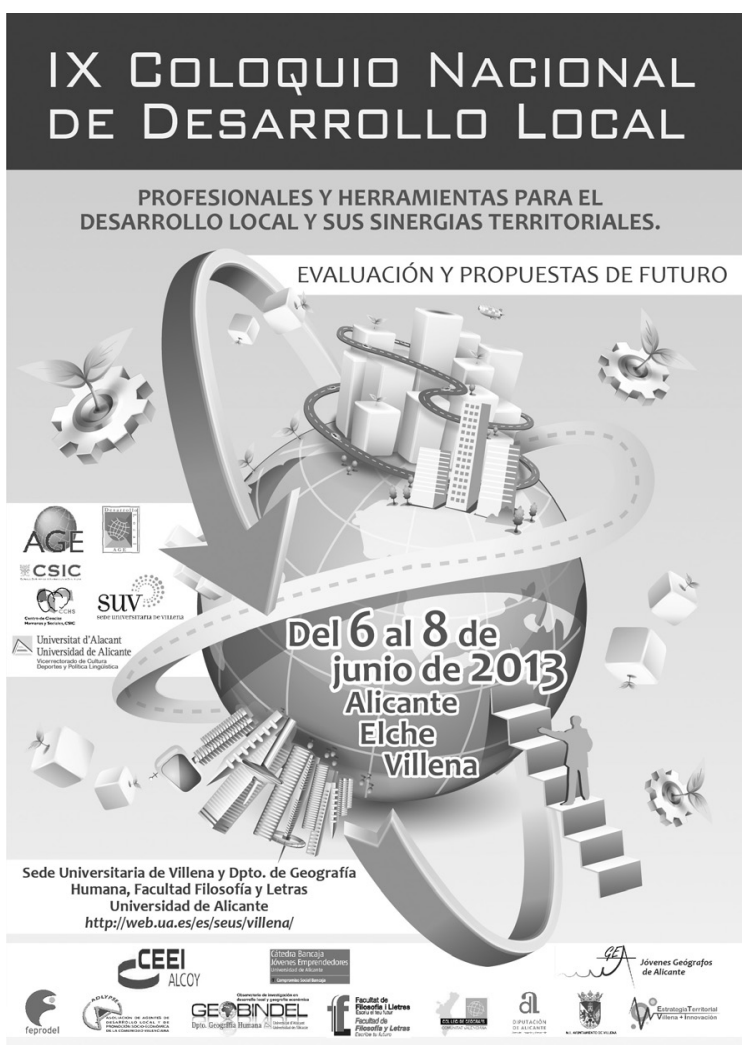


Este libro ha sido examinado y valorado por evaluadores ajenos a la Universidad de Alicante, con el fin de garantizar la calidad científica del mismo.

Publicacions de la Universitat d'Alacant

03690 Sant Vicent del Raspeig

Publicaciones@ua.es

http://publicaciones.ua.es

Telèfon: 965903480

(C) Antonio Martínez Puche, Xavier Amat Montesinos,

Isabel Sancho Carbonell y Daniel Sanchiz Castaño (eds.), 2016

(C) d'aquesta edició: Universitat d'Alacant

ISBN: 978-84-16724-00-0

Dipòsit legal: A 92-2016

Disseny de coberta: candela ink

Composició: Página Maestra (Miguel Ángel Sánchez Hernández)

Impressió i enquadernació: Guada Impresores

\section{unte \\ Unión de Editoriales
Universitarias Españolas \\ WWW.une.es
WWA}

Esta editorial es miembro de la UNE, cosa que garantiza la difusión y comercialización nacional y internacional de sus publicaciones.

Reservados todos los derechos. Cualquier forma de reproducción, distribución, comunicación pública o transformación de esta obra sólo puede ser realizada con la autorización de sus titulares, salvo excepción prevista por la ley. Diríjase a CEDRO (Centro Español de Derechos Repográficos, www.cedro.org) si necesita fotocopias o escanear algún fragmento de esta obra. 


\title{
ALCALÁ DE GUADAÍRA: DE CIUDAD COMPETITIVA A CIUDAD RESILIENTE ${ }^{1}$
}

\author{
Inmaculada Caravaca Barroso \\ Universidad de Sevilla \\ caravaca@us.es \\ Gema González Romero \\ Universidad de Sevilla \\ gemagonzalez@us.es
}

RESUMEN

Resulta de indudable interés profundizar en el conocimiento de la capacidad de respuesta con que cuentan ciertos ámbitos para propiciar el desarrollo y superar los procesos de recesión y crisis, lo que va a depender tanto de sus pautas de comportamiento económico, laboral y social precedentes, como de su grado de dependencia exterior, de la forma en que utilicen sus recursos y de la actitud de sus agentes públicos y privados para hacer frente a los nuevos retos.

En este contexto general de referencia, esta comunicación pretende analizar la incidencia de la crisis en un municipio de la aglomeración urbana de Sevilla, Alcalá de Guadaíra, y su capacidad de reacción ante ella.

Palabras clave: Alcalá de Guadaíra, resiliencia territorial, vulnerabilidad, autoempleo.

\section{ALCALÁ DE GUADAÍRA: FROM COMPETITIVE CITY TO RESILIENT CITY}

\section{Abstract}

Today, it is interesting to know the responsiveness of the territories before the recession and crisis processes, which will depend on both their behaviour

1 Esta comunicación se integra en el Proyecto de $\mathrm{I}+\mathrm{D}+\mathrm{i}$ del Ministerio de Economía y Competitividad, titulado: Efectos socio-territoriales de la crisis económica en las áreas urbanas de España: políticas públicas y estrategias de resiliencia (CSO 2012-36170). 
economic, labor and social precedents, and its degree of external dependence, the use of their resources and the capacity of public and private agents to face new challenges.

In this general context of reference, this paper aims to analyze the impact of the crisis on the city of the urban agglomeration of Sevilla, Alcalá de Guadaíra, and its ability to react to it.

Key words: Alcalá de Guadaíra, territorial resilience, vulnerability, selfemployment.

\section{INTRODUCCIÓN}

Durante las dos últimas décadas del siglo XX fueron numerosos los investigadores que, desde distintas disciplinas, profundizaron en el conocimiento de las intensas transformaciones socioeconómicas que se estaban produciendo y de los impactos territoriales de ellas derivadas. Por una parte, se hacía hincapié en la continua y acelerada incorporación de conocimientos e innovaciones que afectaban de forma muy desigual al dinamismo o declive de los territorios (CASTELLS, 1995 y 1996; GATTO, 1990; MÉNDEZ, 1998 y 2002); por otra, las nuevas tecnologías, fundamentalmente vinculadas a la información y a la comunicación, permitieron la densificación de flujos que conforman el espacio de las redes (VELTZ, 1996 y 1997). A su vez, se revalorizó el rol ejercido por el espacio en los análisis socio-económicos, al considerarlo un importante recurso que contribuye a potenciar o a frenar el desarrollo (SÁNCHEZ, 1988 y 1991; ORTEGA VALCÁRCEL, 1998).

La consideración de estos cambios en la forma de concebir el espacio resulta imprescindible para identificar las formas en que cada territorio se integra en el sistema-mundo, a reconocer cuáles son los territorios ganadores y emergentes y a analizar las claves de su éxito (BENKO Y LIPIEZ, 1994; CARAVACA, 1998).

Estos procesos de transformación socioeconómica y territorial se han profundizado y acelerado en los inicios del siglo XXI, al evidenciarse la existencia de una grave crisis que empezó a detectarse en Estados Unidos en el año 2007, propagándose rápidamente por otros países, y muy especialmente por los de la Unión Europea. Aunque en principio parecía asociada a las actividades financieras e inmobiliarias, se difundió pronto al conjunto de la economía, a la sociedad y a las instituciones, convirtiéndose en sistémica. No puede extrañar, por consiguiente, que sean de nuevo numerosas las investigaciones dedicadas a su análisis (GEORGE, 2010; FERNÁNDEZ DURÁN, 2011; TORRES LÓPEZ, 2011; HARVEY, 2012...).

No obstante, a diferencia de lo ocurrido con la crisis de los años setenta, son ahora muy escasos los estudios que se centran en sus efectos territoriales; y ello pese a que, como señala MÉNDEZ (2013), están provocando impor- 
tantes alteraciones a distintas escalas asociadas al grado de vulnerabilidad de cada ámbito y a su diferente capacidad para desarrollar estrategias con las que superar sus problemas. Esta línea de investigación es, por consiguiente, de indudable interés para la Geografía y trascendental para analizar los procesos de desarrollo local.

En este último sentido, resulta interesante profundizar en el conocimiento de la capacidad con que cuentan ciertos ámbitos para superar los procesos de recesión y crisis, lo que, como es bien sabido, va a depender tanto de sus pautas de comportamiento económico, laboral y social precedentes, como de su grado de dependencia exterior, de la forma en que utilicen sus recursos, de su capacidad para incorporar conocimiento y generar innovaciones y de su habilidad para buscar respuestas colectivas con las que hacer frente a sus problemas (MÉNDEZ, 2002; CARAVACA, GONZÁLEZ-ROMERO, SILVA, 2005; CARAVACA, GONZÁLEZ-ROMERO, 2009).

En este último sentido, la utilización de la categoría conceptual de resiliencia territorial, entendiendo como tal la capacidad mostrada por algunos territorios y ciudades para reaccionar ante la adversidad y hacer frente a sus propios problemas y retos, resulta especialmente adecuada (SIMIE, MARTIN, 2010; CHRISTOPHERSON, MICHIE, TYLER, 2010; MÉNDEZ, 2012; MACKINNON, DRISCOLL-DERICKSON, 2013).

En este contexto general de referencia, esta comunicación pretende analizar la incidencia de la crisis en un municipio de la aglomeración urbana de Sevilla: Alcalá de Guadaíra, y su capacidad de reacción ante situaciones adversas. Si ya en su momento supo adaptarse al proceso de reestructuración industrial vinculado al paso del fordismo al post-fordismo, la grave crisis estructural que actualmente se viene padeciendo le obliga a replantearse cómo hacer frente a las nuevas disfunciones y retos. Se trata, pues, de conocer si esta ciudad media metropolitana está siendo capaz, una vez más, de buscar alternativas de futuro y emprender acciones que le permitan ser no sólo económicamente competitiva, sino también ambientalmente sostenible y socialmente cohesionada, retos que desde hace unos años se había propuesto superar (CARAVACA, GONZÁLEZROMERO, 2010).

Para llevar a cabo esta investigación se han utilizado diversas fuentes estadísticas, así como documentos de planeamiento, informes y estudios. Se han tomado básicamente como fechas de referencia los años 2006 (previo a la crisis), 2009 (crisis iniciada) y 2011/2012 (últimos datos disponibles), aunque se analizan también a veces algunos años previos. Junto a lo anterior, desde hace ya más de una década se ha estado llevando a cabo un sistemático trabajo de campo, basado fundamentalmente en la consulta directa a los agentes locales públicos y privados mediante la realización entrevistas (hasta ahora un total de 75); con esta información se han podido interpretar algunas de las claves que explican la evolución experimentada por Alcalá de Guadaíra. 


\section{La evolución socioeconómica de Alcalá de Guadaíra}

Con una población de 73.675 habitantes (Padrón Municipal de Habitantes de 2012) y ocupando una superficie de $287 \mathrm{~km}^{2}$, el municipio de Alcalá de Guadaíra forma parte de la primera corona metropolitana de la aglomeración urbana de Sevilla. Su participación en el sistema metropolitano determina en gran medida su actual dimensión y la diversificación de su economía, en la que la industria tiene un peso significativo.

La inclusión de parte del término municipal en el Polo de Desarrollo de Sevilla, como estrategia de actuación en materia de planificación regional del Primer Plan de Desarrollo (1964-1967), resultó determinante para que se localizara en él buena parte de las industrias acogidas a sus beneficios, vigentes hasta 1970. Se trataba, sobre todo, de industrias pequeñas y medias, orientadas al consumo y bastante diversificadas, en las que la generación de empleos por inversión fue superior al valor medio establecido en otros Polos. Pese a lo señalado, los resultados económicos del Polo no fueron tan positivos como se esperaba, aunque sí generaron importantes cambios territoriales al alterar la jerarquía urbana provincial, puesto que los dos municipios que, junto a la ciudad de Sevilla, formaban parte del Polo, Dos Hermanas y Alcalá de Guadaíra, pasaron a ocupar desde entonces el segundo y el tercer puesto en el ranking provincial.

Centrando la atención en el ámbito objeto de estudio y como puede apreciarse en el cuadro 1, la población de Alcalá se ha duplicado entre 1960 y 2012, pasando de 30.856 a 73.675 habitantes. Es importante observar que dicho crecimiento se ha producido de forma muy desigual a lo largo del citado periodo. Así, aunque durante los primeros años de vigencia del Polo sólo creció un 8,87 \%, llegó al 35,67\% durante la década de los setenta, poniéndose de manifiesto que los efectos de la política de industrialización fueron más lentos de lo esperado; la reducción del crecimiento al 14,66 \% durante los años ochenta y al 9,89\% en los noventa pueden explicarse por los impactos de los procesos de reestructuración industrial asociados a la crisis del fordismo. Especialmente ilustrativa fue la recuperación experimentada por el crecimiento demográfico entre los años 2001 y 2006 (un 13,17 \%, en sólo cinco años), lo que debe ponerse en relación con las políticas urbanísticas impulsadas por el Ayuntamiento. Pero, a partir de 2006 los efectos de la crisis empiezan a hacerse notar, reduciéndose ligeramente el ritmo de crecimiento de la población entre los años 2006 y 2009 (7,95 \%), y haciéndose ya mucho más patente entre 2009 y $2012(5,02 \%)$ (Cuadro 1). 
Cuadro 1. Evolución de la población de Alcalá de Guadaíra

\begin{tabular}{|l|r|c|c|c|c|c|c|c|}
\hline \multicolumn{1}{|c|}{ Años } & $\mathbf{1 9 6 0}$ & $\mathbf{1 9 7 0}$ & $\mathbf{1 9 8 1}$ & $\mathbf{1 9 9 1}$ & $\mathbf{2 0 0 1}$ & $\mathbf{2 0 0 6}$ & $\mathbf{2 0 0 9}$ & $\mathbf{2 0 1 2}$ \\
\hline $\mathbf{N}^{\mathbf{0}}$ habitantes & 30.856 & 33.593 & 45.577 & 52.257 & 57.426 & 64.990 & 70.155 & 73.675 \\
\hline $\begin{array}{l}\text { Evolución } \\
(\mathbf{1 9 6 0 = 1 0 0 )}\end{array}$ & 100 & 108,87 & 147,71 & 169,36 & 186,11 & 210,62 & 227,36 & 238,77 \\
\hline $\begin{array}{l}\text { Variación entre } \\
\text { períodos \% }\end{array}$ & - & 8,87 & 35,67 & 14,66 & 9,89 & 13,17 & 7,95 & 5,02 \\
\hline
\end{tabular}

Fuentes: Censos de Población y Viviendas y Padrones Municipales de Habitantes, INE.

Si se compara la evolución poblacional relativa de Alcalá de Guadaíra con la de Andalucía, el municipio resulta bastante favorecido (Figura 1). Los procesos de metropolización, junto a la localización en el municipio de un número significativo de industrias, contribuyeron a impulsar su crecimiento demográfico en mucha mayor medida que el del conjunto de la región.

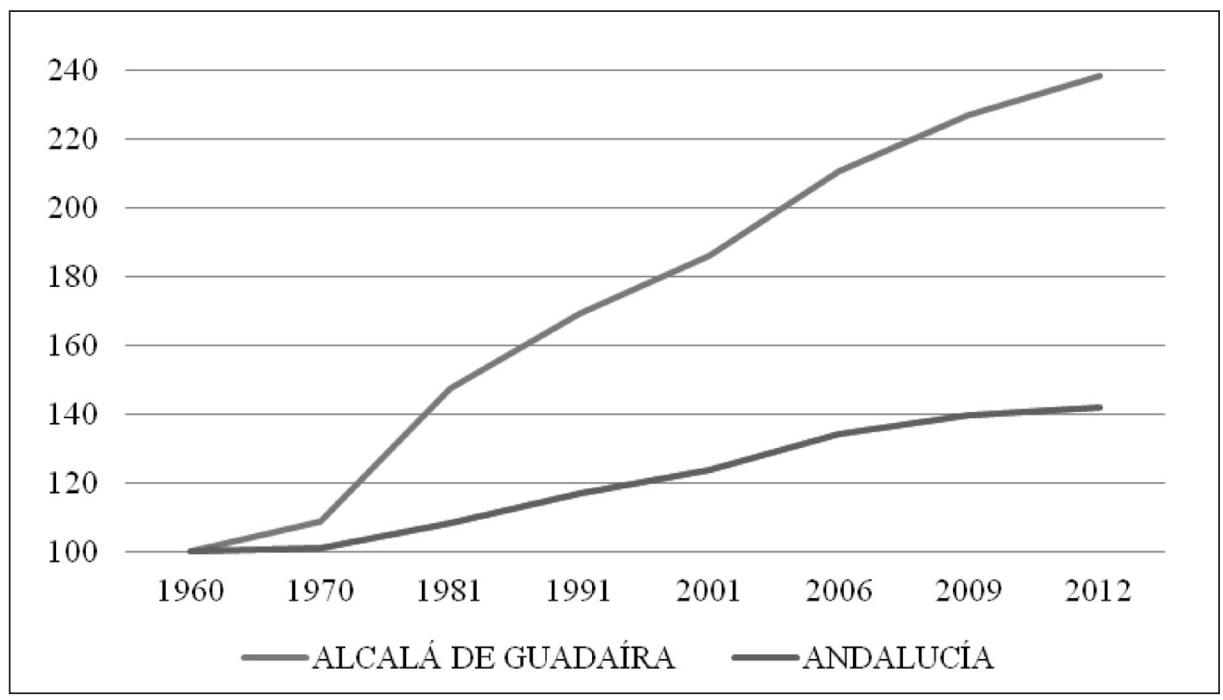

Figura 1. Evolución relativa de la población, 1960-2012 (1960=100).

Fuentes: Censos de Población y Viviendas y Padrón Municipal de Habitantes, INE.

Es especialmente ilustrativa la evolución experimentada por el mercado de trabajo. Como muestra el cuadro 2, el paro registrado no dejó de crecer desde el año 2000, aunque dicho crecimiento se fue acelerando de forma que en 2012 había aumentado un 159,94\%, llegando a representar un 22,18 \% el número de 
parados respecto a la población potencialmente activa ${ }^{2}$. Por su parte, el número de trabajadores, que había aumentado en 9.275 desde el año 2000 al 2006, fue disminuyendo a partir de dicho año perdiéndose 10.540 empleos entre 2006 y 2012, reduciéndose un $41,46 \%$ el número de contratos.

Cuadro 2. Evolución del mercado de trabajo de Alcalá de Guadaíra

\begin{tabular}{|l|r|r|r|r|}
\hline & $\mathbf{2 0 0 0}$ & $\mathbf{2 0 0 6}$ & $\mathbf{2 0 0 9}$ & \multicolumn{1}{c|}{$\mathbf{2 0 1 2}$} \\
\hline Paro registrado & 4.286 & 5.690 & 8.610 & 11.141 \\
\hline Evolución paro registrado $(\mathbf{2 0 0 0 = 1 0 0 )}$ & 100 & 132,76 & 200,89 & 259,94 \\
\hline \% Parados sobre población potencialmente activa & 10,56 & 12,23 & 17,52 & 22,18 \\
\hline $\mathbf{N}^{\mathbf{0}}$ de trabajadores & 19.964 & 29.275 & 25.281 & 18.735 \\
\hline Evolución relativa del $\mathbf{n}^{\mathbf{0}}$ de trabajadores $\mathbf{( 2 0 0 0 = 1 0 0 )}$ & 100 & 146,64 & 126,63 & 93,84 \\
\hline Nuevos contratos & - & 34.938 & 22.149 & 20.452 \\
\hline Evolución nuevos contratos $(\mathbf{2 0 0 6}=\mathbf{1 0 0})$ & - & 100 & 63,40 & 58,54 \\
\hline
\end{tabular}

Fuentes: Padrón Municipal de Habitantes, INE; Trabajadores Afiliados a la Seguridad Social, Tesorería General de la Seguridad Social; Paro Registrado, INEM y SAE; Estadística de Contratos, SAE.

Como complemento de lo anterior, un buen indicador para detectar la incidencia de la crisis en la población puede ser la evolución de sus niveles de renta. No obstante, se trata de un indicador que sólo está disponible a nivel provincial, por lo que se ha utilizado como opción alternativa el Impuesto de la Renta de las Personas Físicas (Agencia Estatal de Administración Tributaria, IECA $)^{3}$. Pese a sus limitaciones, este indicador ayuda a conocer los niveles de riqueza del ámbito objeto de estudio y, en consecuencia, los efectos que en él está causando la crisis. Como puede observarse en el cuadro 3, la evolución interanual experimentada por el IRPF pone de manifiesto que la crisis empieza a notarse en el año 2008, puesto que es en ese año cuando se ralentiza su crecimiento llegando a ser negativo en los dos años siguientes, si bien a partir de 2009 se atenúa esta tendencia. Hasta este último año las cifras correspondientes a Alcalá resultan algo más favorables que las del conjunto de la región, mientras evolucionan peor que las de Andalucía durante el último periodo de referencia.

2 Al no poder contar con cifras municipales recientes de población activa se ha optado por considerar aquellas personas que cuenta entre 16 y 65 años.

3 Esta información cuenta con algunas limitaciones entre las que cabe destacar, por una parte, que no permite detectar las diferencias existentes en el régimen fiscal de los contribuyentes y, por otra, que excluye a las personas exentas de satisfacer este tributo. A su vez, la última información disponible corresponde al año 2010. 
Cuadro 3. Evolución interanual experimentada por el IRPF

\begin{tabular}{|l|c|c|c|c|c|}
\hline & $\mathbf{2 0 0 6}$ & $\mathbf{2 0 0 7}$ & $\mathbf{2 0 0 8}$ & $\mathbf{2 0 0 9}$ & $\mathbf{2 0 1 0}$ \\
\hline Alcalá de G. & 100 & 120,6 & 104,84 & 92,24 & 97,37 \\
\hline Andalucía & 100 & 118,6 & 104,51 & 90,20 & 98,24 \\
\hline
\end{tabular}

Fuente: Agencia Estatal de Administración Tributaria, IECA.

Para completar la información, en la figura 2 se analiza la evolución relativa del consumo eléctrico empresarial y del número de empresas. Las diferencias existentes entre las curvas correspondientes al consumo eléctrico industrial y al del resto de las empresas pone en evidencia que aunque el impacto de la crisis en el sector industrial ha sido hasta 2010 algo más limitado, está creciendo ahora más intensamente.

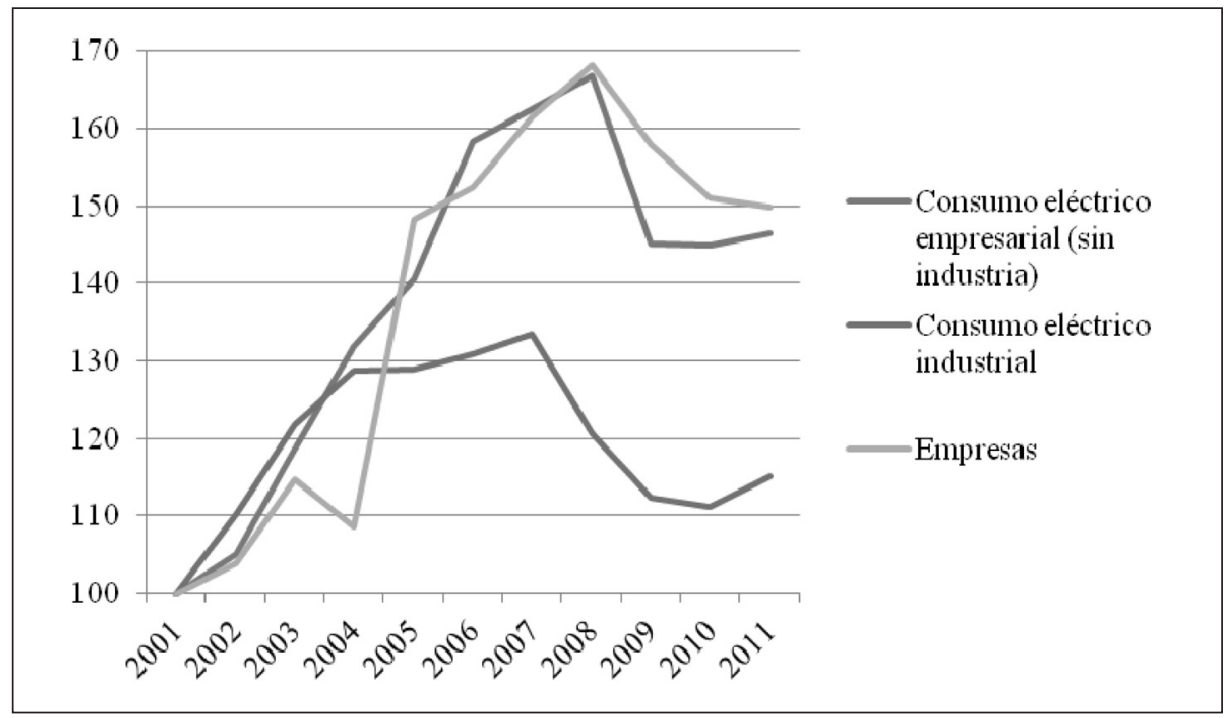

Figura 2. Evolución relativa del consumo eléctrico empresarial (sin industria) e industrial y número de empresas, 2001-2011 (2001=100)

Fuentes: Directorio de Establecimientos con Actividad Económica en Andalucía, IECA; Facturaciones de Endesa Distribución, S.L., IECA

Tal y como muestra el cuadro 4, la reducción del empleo se observa en los cuatro grandes sectores de actividad. Siguiendo la tónica general, tanto en el municipio estudiado como en el conjunto de Andalucía predomina el sector servicios; aunque fue uno de los menos afectados por la crisis entre 2006 y 2009 , la pérdida de puestos de trabajo ha aumentado considerablemente a partir de este último año, lo que está estrechamente relacionado con el fuerte retroce- 
so experimentado por aquellas actividades directamente ligadas al Estado del Bienestar. Muy distinto, sin embargo, es el comportamiento de la industria, con peso muy limitado en la región pero que ocupa en Alcalá la segunda posición en su estructura económica, y es, además, la actividad que menos ha decrecido durante el último periodo analizado; es importante señalar también que, después de Sevilla, Alcalá es el municipio más industrializado de la aglomeración y uno de los más destacados de Andalucía. Como era de esperar, ha sido la construcción el sector más afectado por la crisis tanto en Alcalá como en Andalucía, siendo, a su vez, responsable de la caída del empleo experimentada por aquellas actividades industriales y de servicios ligadas a él (FERNÁNDEZTABALES, CRUZ, 2011).

Cuadro 4. Evolución de la estructura sectorial según número de trabajadores

\begin{tabular}{|c|c|c|c|c|c|c|}
\hline & Agricult. & Industria & Construc. & Servicios & TOTAL \\
\hline \multirow{5}{*}{$\begin{array}{c}\text { Alcalá de } \\
\text { Guadaíra }\end{array}$} & 2006 & $\begin{array}{c}662 \\
(12,26 \%)\end{array}$ & $\begin{array}{c}7.940 \\
(27,12 \%)\end{array}$ & $\begin{array}{c}4.911 \\
(16,77 \%)\end{array}$ & $\begin{array}{c}15.762 \\
(53,84 \%)\end{array}$ & $\begin{array}{c}29.275 \\
(100)\end{array}$ \\
\cline { 2 - 7 } & 2009 & $\begin{array}{c}555 \\
(2,20 \%)\end{array}$ & $\begin{array}{c}7.142 \\
(28,23 \%)\end{array}$ & $\begin{array}{c}3.177 \\
(12,58 \%)\end{array}$ & $\begin{array}{c}14.375 \\
(56,93 \%)\end{array}$ & $\begin{array}{c}25.249 \\
(100)\end{array}$ \\
\cline { 2 - 7 } & $\begin{array}{c}342 \\
(1,82 \%)\end{array}$ & $\begin{array}{c}5.521 \\
(29,47 \%)\end{array}$ & $\begin{array}{c}1.840 \\
(9,8 \%)\end{array}$ & $\begin{array}{c}10.669 \\
(56,94 \%)\end{array}$ & $\begin{array}{c}18.735 \\
(100)\end{array}$ \\
\cline { 2 - 7 } & $2006-2009$ & 83,84 & 89,95 & 64,69 & 91,20 & 86,25 \\
\cline { 2 - 7 } & $\begin{array}{c}2006-2012 \\
(2006=100)\end{array}$ & 51,66 & 69,53 & 37,47 & 67,69 & 64,00 \\
\hline \multirow{5}{*}{ Andalucía } & 2006 & $\begin{array}{c}526.251 \\
(17,29 \%)\end{array}$ & $\begin{array}{c}267.861 \\
(8,80 \%)\end{array}$ & $\begin{array}{c}411.317 \\
(13,51 \%)\end{array}$ & $\begin{array}{c}1.838 .589 \\
(60,40 \%)\end{array}$ & $\begin{array}{c}3.044 .018 \\
(100)\end{array}$ \\
\cline { 2 - 7 } & 2009 & $\begin{array}{c}553.489 \\
(19,64 \%)\end{array}$ & $\begin{array}{c}242.939 \\
(8,62 \%)\end{array}$ & $\begin{array}{c}217.904 \\
(7,73 \%)\end{array}$ & $\begin{array}{c}1.803 .066 \\
(64,00 \%)\end{array}$ & $\begin{array}{c}2.817 .398 \\
(100)\end{array}$ \\
\cline { 2 - 7 } & $\begin{array}{c}244.485 \\
(12,98 \%)\end{array}$ & $\begin{array}{c}179.539 \\
(9,54 \%)\end{array}$ & $\begin{array}{c}89.953 \\
(4,78 \%)\end{array}$ & $\begin{array}{c}1.368 .478 \\
(72,69 \%)\end{array}$ & $\begin{array}{c}1.882 .455 \\
(100)\end{array}$ \\
\cline { 2 - 7 } & $\begin{array}{c}2006-2009 \\
(2006=100)\end{array}$ & 105,18 & 90,70 & 52,98 & 98,07 & 92,56 \\
\cline { 2 - 7 } & $\begin{array}{c}2006-2012 \\
(2006=100)\end{array}$ & 46,46 & 67,03 & 21,87 & 74,43 & 61,84 \\
\hline
\end{tabular}

Fuente: Empresas y Trabajadores Afiliados a la Seguridad Social, Tesorería General de la Seguridad Social. 


\section{Estrategias y aCtUACIONES PÚBLICAS LOCALES}

Existen diferencias significativas en los niveles de desarrollo de ámbitos geográficamente cercanos y dotados de recursos similares, siendo aquéllos que han sido capaces de activarlos y ponerlos en valor los que suelen estar más articulados socialmente y los que están llevando a cabo proyectos comunes que favorecen no sólo la competitividad económica sino también la cohesión social y el desarrollo territorial.

En el caso concreto que nos ocupa, entre las estrategias públicas locales desarrolladas durante las últimas décadas, destaca la vinculada al proyecto $A l$ calá Reserva Industrial de Andalucía, ARIA (RIBERA, 1995), que centraba la atención en la revitalización de la industria y en la rehabilitación de la elevada superficie de suelo empresarial existente en el municipio, buena parte del mismo creado como apoyo infraestructural al Polo de Desarrollo. Con el citado proyecto, desarrollado por la corporación municipal, se iniciaba una etapa en la que no sólo se centraba la atención en actuaciones de rehabilitación y mejora, sino que suponía una nueva concepción de este tipo de suelo, considerándolo un importante recurso para potenciar el desarrollo empresarial (CARAVACA et AL, 2009).

Con esta actuación se iniciaba una serie de estrategias que desde entonces se vienen implementando y que se sustentan en la creación de una serie de organismos e infraestructuras para la promoción del desarrollo y la innovación que se llevan a cabo a través de la Agencia Municipal de Desarrollo. Entre sus actuaciones es necesario destacar la creación del Complejo de Innovación y Desarrollo de Alcalá de Guadaíra (IDEAL) en el que, junto a los organismos e infraestructuras dependientes del Ayuntamiento, se ubican algunos otros, como es el caso, por ejemplo, de la Cámara de Comercio. La figura 3 intenta sistematizar los promovidos desde el Ayuntamiento.

Entre las infraestructuras que sirven de base a la promoción económica se incluyen el Centro de Exposiciones y Congresos, una incubadora de empresas que presta especial atención a los proyectos de base tecnológica, el Centro de Apoyo a la Calidad y la Seguridad en la Industria, y el Centro de Realidad Virtual. Por su parte, entre los organismos que no sólo apoyan la promoción económica sino también la innovación, destaca con mucho la Sociedad Innovar en Alcalá, de capital municipal, que, además de gestionar el Complejo de Innovación y Desarrollo antes mencionado, se encarga de asesorar y tutelar a las empresas; desde 2012 incluye entre sus objetivos propiciar la sostenibilidad económica y ambiental, la creación de empresas en sectores considerados emergentes en Andalucía y la rehabilitación urbanística de áreas residenciales, industriales y logísticas. A la anterior se suma también la Fundación Alcalá Innova, de capital mixto. Especial mención merece también el Centro de Formación Avanzada, integrado en la Agencia Municipal de Desarrollo, que organiza cursos y seminarios para empresarios y trabajadores en colaboración con la Universidad Pablo de Olavide (Figura 3). 


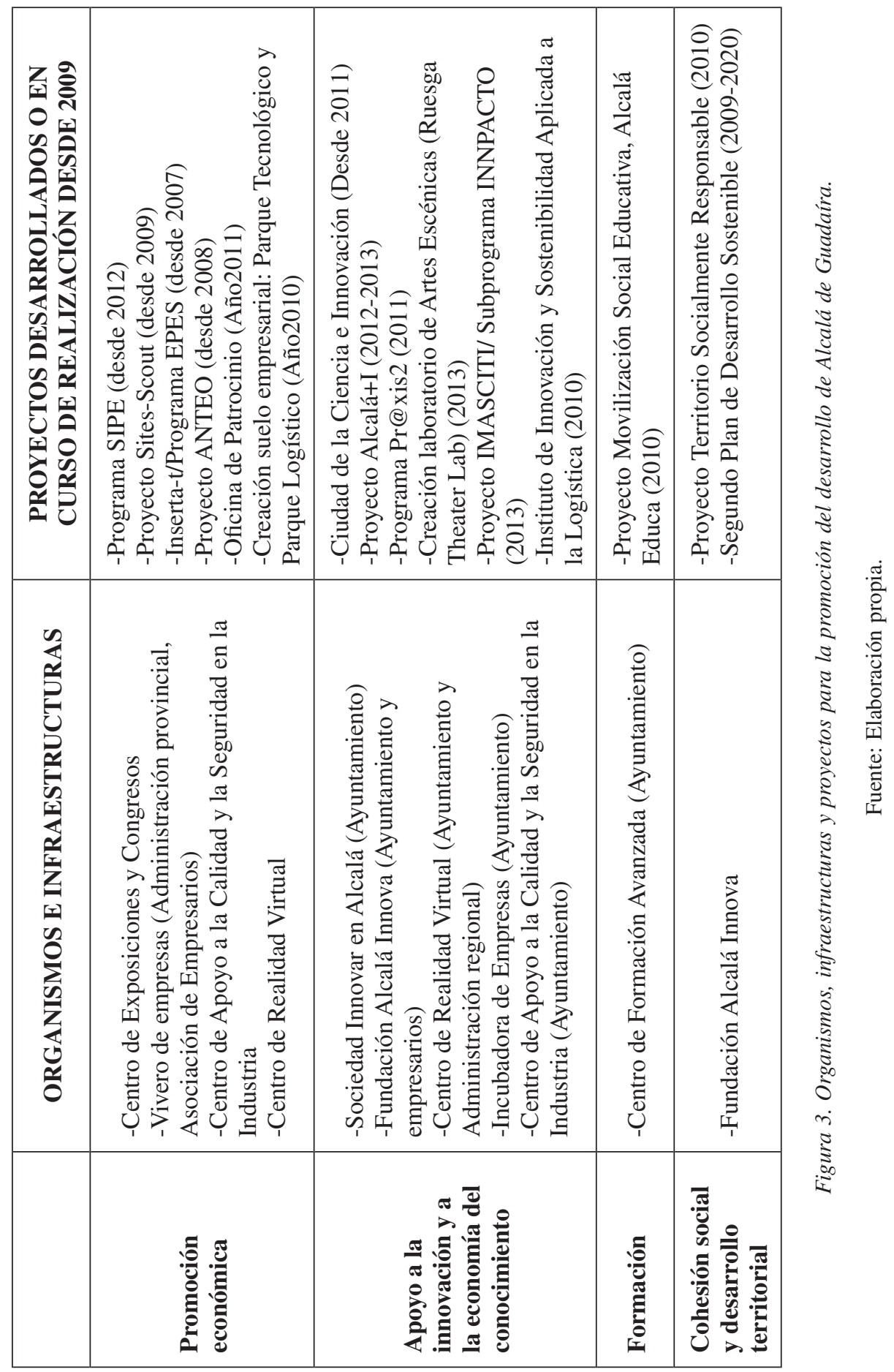


Para poder comprobar la capacidad de resiliencia que Alcalá de Guadaíra está demostrado ante la crisis, resulta de especial interés el análisis de los proyectos que se han concebido y aprobado una vez iniciada la misma.

La promoción de nuevos empleos se está llevando a cabo, fundamentalmente, a partir de dos tipos estrategias: el autoempleo, y la inserción y recualificación profesional de los desempleados. Dentro de la primera hay que enmarcar el Proyecto de Simulación de Empresas para Emprendedores (SIPE), una iniciativa del Ministerio de Industria, Energía y Turismo que se está implementando en Alcalá a partir de la Sociedad para la Promoción del Desarrollo y el Turismo de la Diputación de Sevilla y el propio Ayuntamiento para intentar impulsar el emprendimiento. La segunda de las estrategias se está llevando a cabo a través de diferentes actuaciones que, siendo alentadas por la corporación local, cuentan con financiación externa proveniente del Fondo Social Europeo y del gobierno regional. Una de ellas es Sites-Scout (Sistema de Información Territorial para el Empleo Social) que, implementada por el Ayuntamiento en colaboración con otras ciudades europeas (Roma y Pescaria en Italia, Asmoune en Francia y Manlleu en España), persigue mejorar el grado de formación e inserción laboral de los colectivos más desfavorecidos. Además, el proyecto Inserta-t, perteneciente al Programa de Experiencias Profesionales para el Empleo de la Junta de Andalucía, está dirigido a la inserción de las personas cualificadas en situación de desempleo mediante la realización de prácticas en empresas.

Por su parte, la Fundación Alcalá Innova ha puesto en marcha diferentes talleres centrados en las posibles estrategias y respuestas que las PYMEs pueden desarrollar frente a la crisis. En este sentido, el Proyecto ANTEO contribuye a fomentar la cooperación y los intercambios entre las PYMES de Alcalá de Guadaíra. A su vez, el Ayuntamiento ha creado la Oficina de Patrocinio con objeto de impulsar la implicación de las empresas en las diferentes acciones de promoción económica y desarrollo que realizan los organismos públicos.

A todas estas actuaciones hay que sumar las dirigidas a ampliar la oferta y calidad de suelo productivo. Así se explica que, junto a las actuaciones de rehabilitación del suelo empresarial, se estén ejecutando nuevos proyectos como un parque tecnológico y otro logístico.

Pero, para impulsar la competitividad empresarial se está haciendo especial hincapié en la incorporación de innovaciones. Un reconocimiento del esfuerzo que los actores públicos locales han realizado en ese sentido es que Alcalá de Guadaíra ostenta desde el año 2011 el distintivo de Ciudad de la Ciencia e Innovación, otorgado por el entonces Ministerio de Ciencia e Innovación (ahora de Economía y Competitividad), que la reconoce como una de las ciudades líderes en la creación de ventajas competitivas. Esta distinción hace que Alcalá forme parte de la denominada Red Impulso, foro de encuentro nacional en el que se definen políticas locales innovadoras. 
El Ayuntamiento, en colaboración con el Ministerio de Economía y Competitividad, ha aprobado el Proyecto Alcalá $+I$ que, financiado por el Fondo Tecnológico FEDER, busca mejorar la competitividad de las PYMES a partir del esfuerzo y la atención constante a la innovación. También se enmarca en esta línea elProgramaPr@xis2, desarrollado por la Diputación Provincial en colaboración con el Ayuntamiento, que tiene como objetivo lograr un mayor desarrollo de los servicios avanzados a las empresas.

La administración local, apostando por aquellos sectores que pudieran contribuir a cambiar el modelo productivo, ha creado el Laboratorio de Artes Escénicas, que pretende convertir al municipio en un distrito creativo, favoreciendo para ello la investigación, el desarrollo, la innovación, el asesoramiento y la interrelación de las diferentes actividades ligadas a las artes escénicas.

De diferente índole es el Proyecto IMASCITI, que busca desarrollar la gobernanza mediante el uso de nuevas tecnologías de comunicación que permitan mayor eficiencia de los servicios que presta la administración local, así como potenciar la colaboración ciudadana. En este caso, esta ciudad junto con Burgos, se convertirá en 2014 en una de las primeras ciudades inteligentes, denominación que utiliza el Ministerio de Economía y Competitividad para las que participan en el Subprograma INNPACTO.

Por su parte, el Instituto de Innovación y Sostenibilidad Aplicada a la Logística, dedicado al fomento de estas actividades, pretende ser el primer soporte del parque logístico proyectado, que está incluido en el Plan de Ordenación del Territorio de Aglomeración Urbana (POTAU) como Área de Oportunidad.

Con el objetivo de propiciar el interés por el aprendizaje y el conocimiento se desarrolla el Proyecto Movilización Social Educativa Alcalá Educa que, además de contar con una línea de sensibilización de la cultura emprendedora, pretende reducir el fracaso escolar, educar en valores y fomentar el interés por el aprendizaje. En esta línea, se explica la integración de Alcalá en la Red Internacional de Ciudades Educadoras lo que, además de contribuir a canalizar información e innovaciones desde el exterior, parece responder también a las nuevas estrategias de gobierno del territorio.

Desde la perspectiva socio-territorial requiere así mismo atención el Proyecto Territorio Socialmente Responsable, que se está llevando a cabo en colaboración con la Organización Internacional del Trabajo. En él participan asociaciones empresariales y sociales, empresas, sindicatos, universidades y la administración regional; su objetivo es implicar a la sociedad local en un proyecto de ciudad económicamente dinámica, ambientalmente sostenible y socialmente cohesionada.

Por último, entre 1998-2008 se desarrolló el Primer Plan de Desarrollo Sostenible que, implementado en un período de bonanza económica, tuvo como principales objetivos: construir una identidad territorial propia y consolidar un tejido productivo dinámico e innovador. Actualmente se está redactando un 
Segundo Plan de Desarrollo Sostenible 2009-2020, que pretende adecuarse a las nuevas realidades socioeconómicas y territoriales.

En general, las estrategias de desarrollo llevadas a cabo por el Ayuntamiento de Alcalá de Guadaíra se centran, pues, en tres pilares: la innovación empresarial, la educación como base del conocimiento y la responsabilidad social. Se trata, por consiguiente, de una interesante apuesta que pone en evidencia la capacidad de las administraciones locales para diseñar estrategias que permitan poner en valor los recursos intangibles y que propicien un cambio en la lógica de funcionamiento económico.

El escaso intervalo de tiempo desde la formulación de estas estrategias y el hecho de que estén coincidiendo con la crisis económica impide aún hacer un balance de los resultados obtenidos, pero el propio diseño de las mismas y los conceptos de los que parten deben ser reconocidos como un valor; sobre todo teniendo en cuenta que buena parte de las medidas adoptadas desde el inicio de la crisis pueden ser consideradas como proactivas y pretenden superar una etapa especialmente adversa.

\section{Algunas Conclusiones}

Alcalá de Guadaíra es una de las ciudades medias andaluzas más potentes económicamente. Su carácter metropolitano y el hecho de situarse en uno de los principales ejes de articulación regional son dos de los factores explicativos de tal situación.

Es un municipio joven, con una dinámica demográfica y migratoria positiva, que cuenta con efectivos poblacionales suficientes para el desarrollo de la actividad económica. Sin embargo, sus niveles de formación son, por lo general, bajos y el mercado laboral no logra satisfacer la totalidad de las demandas de puestos de trabajo, adoleciendo además los empleos de cierta precariedad.

La economía alcalareña se caracteriza por el importante papel que en ella ejerce la actividad industrial. El carácter emprendedor de su clase empresarial y la diversificación de la industria municipal son dos de sus principales puntos fuertes, a los que hay que añadir su capacidad de atracción de empresas exógenas, dada su localización en la primera corona metropolitana. No obstante, es necesario tener en cuenta que estas ventajas pueden verse ensombrecidas por el hecho de que la mayoría de sus actividades son de intensidad tecnológica media, de tal suerte que pueden verse amenazadas tanto por la competencia en costos de terceros países como por las mejores condiciones tecnológicas de otros ámbitos.

Las condiciones de su suelo empresarial son uno de los factores a tener en cuenta en el futuro desarrollo económico del municipio. A pesar de la creación de nuevos espacios productivos mejor dotados, y de los esfuerzos realizados en la rehabilitación de zonas y polígonos industriales desarrollados en etapas 
anteriores, la presencia de suelo empresarial obsoleto y en mal estado puede mermar la competitividad y el atractivo del municipio como lugar de inversión de nuevas industrias.

Son muchas y diversas las estrategias y actuaciones llevadas a cabo por la corporación municipal para impulsar el desarrollo, potenciar la capacidad innovadora y contribuir a poner en valor el conocimiento como base indispensable para el desarrollo. Si bien es cierto que algunas de ellas se vienen desarrollando desde principios de siglo, también lo es que se han potenciado durante los últimos años buscando reaccionar frente a los efectos negativos de la crisis.

El rasgo más destacable de las actuaciones públicas locales que se están llevando a cabo en Alcalá de Guadaíra es que buena parte de ellas se realizan en colaboración, ya sea con las administraciones nacional, regional y provincial, o con otros organismos de carácter privado o mixto de otros ámbitos nacionales o extranjeros. Esto tiene el interés añadido de integrar al municipio en redes, ya sean éstas internas o externas.

En resumidas cuentas, puede decirse que Alcalá de Guadaíra está trabajando activamente para lograr avanzar en su proceso de desarrollo territorial, contribuyendo a potenciar tanto la capacidad competitiva e innovadora de las empresas como el bienestar de sus habitantes. Las numerosas actuaciones implementadas por la Corporación Municipal durante las últimas décadas, se han multiplicado tras el inicio de la crisis, abordando aspectos no sólo ligados a la competitividad económica, sino también a la cohesión social y al desarrollo territorial. De los resultados derivados de tales actuaciones en pro de la resolución y superación de la crisis dependerá su condición de ciudad resiliente. Hasta el momento, la actitud diligente de los actores locales y la profusión de medidas adoptadas en tal sentido muestra su implicación y empeño en conseguirlo.

\section{BIBLIOGRAFÍA}

BENKO, G. - LIPIETZ, A. (1994): Las regiones que ganan. Valencia, Ediciones Alfons el Magnánim. Feneralitat Valenciana y Diputación Provincial.

CARAVACA, I. (1998): "Los nuevos espacios ganadores y emergentes" EURE. Revista Latinoamericana de Estudios Urbano-Regionales, Vol XXIV, $\mathrm{n}^{\circ} 73$, 1998, pp. 5-30.

CARAVACA, I. - GONZÁLEZ-ROMERO, G. - SILVA, R. (2005): "Innovación, redes, recursos patrimoniales y desarrollo territorial" EURE Revista Latinoamericana de Estudios Urbano Regionales, VOL.XXXI, Nº. 94, pp. 5-24.

CARAVACA, I. - GONZÁlEZ-ROMERO, G. - MENDOZA, A. - SILVA, R. (2009): Dinamismo, innovación y desarrollo en ciudades pequeñas y medias de Andalucía, Sevilla, Consejo Económico y Social de Andalucía Premio de Investigación 2008. 
CARAVACA, I.- GONZÁLEZ-ROMERO, G. (2009): "Las redes de colaboración como base del desarrollo territorial", Scripta Nova, vol. XII, $N^{o} 289$, http://www.ub.es/geocrit/sn/sn-289.htm.

CARAVACA, I.- GONZÁLEZ-ROMERO, G. (2010): "Estrategias y actuaciones para el desarrollo en ciudades medias. Algunos ejemplos" Scripta Nova, vol. XIII, http://www.ub.es/geocrit/sn/sn-344htm.

CASTELLS, M. (1995): La ciudad informacional. Madrid. Alianza.

CASTELLS, M. (1996): The rise of the Network Society. MassachussettsOxford, Blakwell.

CHRISTOPHERSON, S. - MICHIE, J. - TYLER, P. (2010): "Regionasl resilience: theoretical and empirical perspectives" Cambridge Journal of Regions, Economy and Society, no 3. pp. 3-10.

FERNÁNDEZ DURÁN, R. (2011): La quiebra del capitalismo global: 20002030. Bilbao, Virus Editorial.

FERNANDEZ-TABALES, A. y CRUZ, E. (2011): “Territorio y actividad constructora: del "tsunami" a la crisis. Factores explicativos y propuesta de indicadores a escala municipal en Andalucía". Boletín de la Asociación de Geógrafos Españoles, n. 56, pp. 79-110.

GATTO, F. (1990): “Cambio tecnológico neofordista y reorganización productiva. Primeras reflexiones sobre sus implicaciones territoriales", en Alburquerque, F. Coord. Revaluación tecnológica y reestructuración productiva. Impactos y dasafíos territoriales. Buenos Aires, Grupo Editor Latinoamericano, pp. 55-102.

GEORGE, S. (2010): Sus crisis, nuestras soluciones. Barcelona Icaria-Intermón-Oxfam.

HARVEY, D. (2012): El enigma del capital y las crisis del capitalismo. Madrid, Akal.

MACKINNON, D. y DRISCOLL DERICKSON, K. (2013): "From resilience to resourcefulness: A critique of resilience policy and activism”. Progress in Human Geography, 37, pp. 253-270.

MÉNDEZ, R. (1998): “Innovación tecnológica y reorganización del espacio industrial: una propuesta metodológica”. EURE, Revista Latinoamericana de Estudios Urbanos Regionales, 24, 73: 31-54.

MÉNDEZ, R. (2002): "Innovación y desarrollo territorial: Algunos debates teóricos recientes". EURE, Revista Latinoamericana de Estudios Urbanos Regionales 28, 84: 63-84.

MÉNDEZ, R. (2012): “Ciudades y metáforas: sobre el concepto de resiliencia urbana”. Ciudad y Territorio. Estudios Territoriales. Vol. XLIV, n 172, pp. 215-232.

MÉNDEZ, R. (2013): Las escalas de la crisis. Ciudades y desempleo en Espa$\tilde{n} a$, Fundación $1^{\circ}$ de Mayo, Colección Estudios www.1mayo.cooo.es. 
ORTEGA VALCÁRCEL, J. (1998): "El patrimonio territorial como recurso cultural y económico" Ciudades 4. Territorio y Patrimonio, Valladolid, Instituto de Urbanística de la Universidad de Valladolid.

RIBERA, L. Dir. (1995): Alcalá Reserva Industrial de Andalucía, Documento inédito.

SÁNCHEZ, J. E. (1988): "Espacio y NuevasTecnologías". Geocrítica. no 78.

SÁNCHEZ, J. E. (1991): Espacio, economía y sociedad. Madrid, Siglo XXI.

SIMIE, J.-MARTIN, R. (2010): "The economic resilience of regiones:towards an evolutionary approach", Cambridge Journal of Regions, Economy and Society, $n^{\circ}$ 3. pp. $27-43$

TORRES LÓPEZ, J. (2011): Contra la crisis, otra economía y otro modo de vivir. Móstoles (Madrid), Ed. HOAC.

VELTZ, P. (1996): Mondialisation villes et Territoires. L'Economica d'archipel. Paris. P.U.F.

VELTZ, P. (1997): "L'économie mondiale, une économicie d'archipel" en Cordellier, S. Doutant, F. Coords. Mondialisation an des mythes. pp. 59-67. 